Review Article

\title{
Meta-Analysis Based on Clinical RCTs: The Effect of Molecular Epimerism on the Safety of Glycyrrhizic Acid
}

\author{
Hongdou Chen, ${ }^{1,2}$ Fangfang Zheng, ${ }^{1,2}$ Menglei Wang $\mathbb{D}^{1,2}$ Xu Wang, ${ }^{1,2}$ Qingqing Yang, ${ }^{1,2}$ \\ Lu Ye, ${ }^{1,2}$ Yao Lu, ${ }^{1,2}$ Shule Yu, ${ }^{1,2}$ and Wei $\mathrm{Li}^{1,2}$ \\ ${ }^{1}$ Department of Pharmacy, Suqian People's Hospital of Nanjing Drum Tower Hospital Group, Suqian 223800, Jiangsu, China \\ ${ }^{2}$ Department of Pharmacology, Suqian Hospital Affiliated with Xuzhou Medical University, Suqian 223800, Jiangsu, China
}

Correspondence should be addressed to Menglei Wang; 77107301@qq.com

Hongdou Chen and Fangfang Zheng contributed equally to this work.

Received 13 May 2020; Revised 30 August 2020; Accepted 10 November 2020; Published 30 November 2020

Academic Editor: Armando Zarrelli

Copyright (c) 2020 Hongdou Chen et al. This is an open access article distributed under the Creative Commons Attribution License, which permits unrestricted use, distribution, and reproduction in any medium, provided the original work is properly cited.

Objective. To carry out the meta-analysis on the clinical safety of glycyrrhizic acid and the influencing factors between $18 \alpha$ glycyrrhizinate $(18 \alpha-\mathrm{GL})$ and $18 \beta$-glycyrrhizinate $(18 \beta-\mathrm{GL})$. Methods. Magnesium isoglycyrrhizinate injection was used as the representative preparation of $18 \alpha-\mathrm{GL}$, and compound glycyrrhizin injection was used as the representative preparation of $18 \beta$-GL. The clinical control trial of magnesium isoglycyrrhizinate injection and compound glycyrrhizin injection was searched in a computer, which was published from January 2006 to December 2019 on the databases such as PubMed, China National Knowledge Infrastructure (CNKI), China Science and Technology Journal Database (CSTJ), and Wanfang Medical Network (Wanfang Data). The data associated with adverse drug reactions (ADRs) were extracted. RevMan5.3 was used for statistical analysis. Results. Finally, 24 studies were included, and 2757 patients were involved, of which the experimental group was mainly treated with magnesium isoglycyrrhizinate, while the control group was mainly treated with compound glycyrrhizin. The results showed that the occurrence of ADRs was significantly lower in the experimental group than that in the control group, and the difference between two groups was statistically significant $(\mathrm{RR}=0.26,95 \% \mathrm{CI}=(0.18,0.38), P<0.00001)$. There was no heterogeneity among the studies $\left(I^{2}=0 \%, P=1.00\right)$. Conclusion. Compared with $18 \beta-\mathrm{GL}, 18 \alpha$-GL had a lower incidence of adverse reactions and better clinical safety.

\section{Introduction}

Glycyrrhizin (GL) is one of the active ingredients in licorice, and it has been widely used in clinical diseases of abnormal liver function. Based on epimerism, GL is classified into $18 \alpha$-glycyrrhizinate $(18 \alpha-\mathrm{GL})$ and $18 \beta$ glycyrrhizinate $(18 \beta-\mathrm{GL})$ [1]. The difference between the efficacy and safety of these two epimers has become the focus of clinical attention. Currently, the representative $18 \alpha$-GL drug on the market is magnesium isoglycyrrhizinate (MI), and the representative $18 \beta-\mathrm{GL}$ drug is compound glycyrrhizin (CG). Existing studies with small samples have shown that MI and CG have differing conclusions of the adverse drug reactions (ADRs). The studies of Ye et al. [2] and Mao et al. [3] showed that there were no differences in ADRs between these two drugs. Many other controlled studies only reported the number of ADR cases from these two drugs and did not perform statistical analyses. Animal studies have shown that the two isomers are different in cortisol competition for $11 \beta$ hydroxysteroid dehydrogenase [4]; conversely, $11 \beta$ hydroxysteroid dehydrogenase was directly associated with pseudo-aldosteronism-related symptoms. However, whether the difference in the structure of these two preparations has led to the occurrence of ADR difference has not been reported clinically. Therefore, in this study, 
meta-analysis was used to explore their relationship of ADRs and 11 $\beta$-hydroxysteroid dehydrogenase in MI and CG in order to provide reference for clinical safe medication and new production preparations.

\section{Materials and Methods}

2.1. Literature Retrieval. With the keywords of MI and CG, the literature from January 2006 to December 2019 was searched in the databases such as PubMed, China National Knowledge Infrastructure (CNKI), China Science and Technology Journal Database (CSTJ), and Wanfang Medical Network (Wanfang Data). And then, the references of the literature that met the inclusion criteria were carefully read to obtain the wanted articles. The selected literature was published in Chinese or English. This paper was retrieved by two researchers (Fangfang Zheng and Xu Wang).

\subsection{Selection Criteria}

\subsubsection{Literature Inclusion Criteria}

(1) The subjects were patients with abnormal liver biochemical indicators

(2) The case-control study on the experimental group treatment with MI injection and control group treatment with CG injection and other treatment measures in these two groups which were the same in patients with abnormal liver function was analyzed

(3) These articles reported in detail the number and presentation of ADRs in the experimental and control groups

\subsubsection{Literature Exclusion Criteria}

(1) The same article is in different databases

(2) Different articles using the same data repeatedly

(3) ADR data are in doubt

2.3. Literature Screening. Two researchers (Lu Ye and Qingqing Yang) extracted the papers. First of all, through reading the title and abstract of the literature retrieved, articles of magnesium isoglycyrrhizinate and compound glycyrrhizin were screened out. Articles that remained after the preliminary screening were screened again by reading the entire articles based on the provided ADR data. Finally, data extracted from all articles were compared to remove duplicate articles. Articles that only reported the number or the presentation of ADRs or articles in which the reported number did not match with the presentation were excluded.

2.4. Data Extraction and Quality Evaluation. All the following information was extracted from the included literature: diagnosis, diagnosis and treatment cycle, MI dose (mg), MI origin, CG dose (mg), CG origin, MI sample size, CG sample size, MI-ADR number and presentation, and CG-ADR number and presentation. The quality of the literature was evaluated according to the Cochrane risk-of-bias tool [5]. Lowquality or missed information literature studies were removed. Two evaluators evaluated the literature independently and cross-checked. When there were divergences, the consensus was reached through discussion.

2.5. Statistical Analysis. A meta-analysis was carried out by RevMan5.3 software. The publication bias was tested by Stata software. The development of ADRs was evaluated using the relative risk (RR) and 95\% confidence interval (CI). Heterogeneity was examined using a homogeneity test (chisquare test). $I^{2} \leq 50 \%$ indicated that the heterogeneity might not be important or that there was moderate heterogeneity. The fixed-effect model was used for the meta-analysis. $I^{2}$ $>50 \%$ indicated that heterogeneity was evident. If interference could not be excluded, the random-effect model was used for analysis.

\section{Results}

3.1. Literature Retrieval Results. Databases were searched comprehensively, and then, the retrieval results were crosschecked (Figure 1). At the end, 55 articles were chosen for the meta-analysis. Preliminary screening was performed on the titles and abstracts, and two non-RCTs $[6,7]$ and two articles with nonmatched drugs were removed [8, 9]. After reading the entire articles, one article with repeated data [10], one retrospective study [11], 20 articles that did not report ADR data [12-31], three articles that only reported the number of ADRs but did not report symptoms [32-34], and two articles that had inconsistent ADR numbers and symptoms were excluded [35, 36]. Finally, 24 articles that conformed to the inclusion criteria were included in this study [3, 37-59].

These 24 articles involved a total of 2,757 patients with 1,520 cases in the MI group and 1,237 cases in the CG group. The MI doses in these studies ranged from 50 to $200 \mathrm{mg}$, and the CG doses ranged from 60 to $200 \mathrm{mg}$. The MI preparations in these studies were from original manufacturers. Nine studies had labeled the origins of the CG injections, and five studies did not indicate the origin; therefore, a statistical analysis could not be performed (Table 1).

3.2. Evaluation of Literature Quality and the Heterogeneity Test. Three $[37,38,57]$ of the 24 articles described the method of randomization. None of the articles mentioned allocation concealment. One article used a double-blinded method. One article was published in a Chinese-English journal [3], and the other articles were all published in Chinese journals. The determination of ADRs was based on clinical symptoms and laboratory biochemical indicators; therefore, it was objective. Data in all articles were complete, and there was no loss to follow-up. Because most of the articles were published in Chinese journals, publication bias was evaluated using a funnel plot. The results are shown in Figure 2. The funnel plot was tested by Begg's test (Figure 3 ). The results showed that $z=0.64$ (continuity corrected), $\mathrm{pr}>|Z|=0.519$ (continuity corrected), 


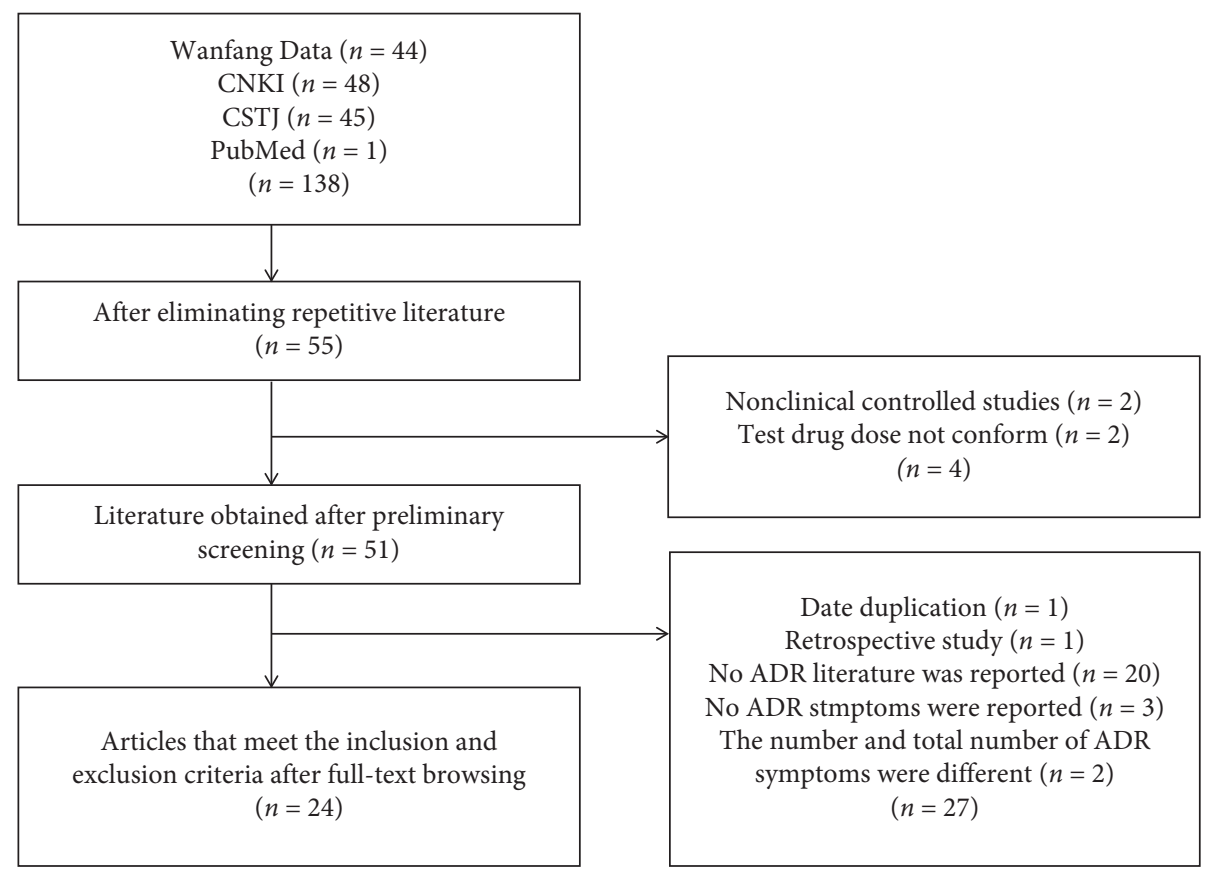

Figure 1: Flow diagram of assessment of studies.

TABle 1: Basic information of included studies.

\begin{tabular}{|c|c|c|c|c|c|c|c|}
\hline \multirow{2}{*}{ Study } & \multicolumn{3}{|c|}{ MI } & \multicolumn{3}{|c|}{ CG } & \multirow{2}{*}{ SI } \\
\hline & Number & Dose (mg) & $\mathrm{ADR}$ & Number & Dose $(\mathrm{ml})$ & $\mathrm{ADR}$ & \\
\hline Ao, 2018 & 50 & 100 & 1 & 50 & 80 & 3 & (3) (4) (5) \\
\hline Cai, 2012 & 32 & 150 & 0 & 30 & 60 & 1 & (1) (2) (3) (4) (5) \\
\hline Chen, 2015 & 40 & 150 & 0 & 40 & 80 & 2 & (1) (2) (3) (4) (5) \\
\hline Cui, 2016 & 50 & 150 & 1 & 50 & 80 & 13 & (4) (5) \\
\hline $\mathrm{Hu}, 2014$ & 30 & 100 & 0 & 30 & 100 & 2 & (1) (2) (3) (4) (5) \\
\hline Li, 2009 & 26 & 100 & 1 & 30 & 60 & 7 & (4) (5) \\
\hline $\mathrm{Li}, 2010$ & 30 & 100 & 0 & 28 & 40 & 2 & (1) (2) (3) (4) (5) \\
\hline Liu, 2017 & 54 & 150 & 0 & 54 & 80 & 2 & (1) (2) (3) (4) (5) \\
\hline Mao, 2009 & 360 & $100-150$ & 5 & 120 & 60 & 6 & (1) (2) (4) (5) \\
\hline Mo, 2012 & 84 & $100-200$ & 0 & 84 & $40-60$ & 2 & (1) (2) (3) (4) (5) \\
\hline Shang, 2013 & 40 & 200 & 0 & 40 & 100 & 4 & (1) (2) (3) (4) \\
\hline Shen, 2012 & 50 & 200 & 3 & 50 & 80 & 9 & (1) (3) (4) (5) \\
\hline Tan, 2011 & 28 & 100 & 2 & 27 & 80 & 3 & (1) (2) (3) (4) \\
\hline Wang, 2013 & 54 & 150 & 4 & 61 & 80 & 13 & (1) (2) (4) \\
\hline Wang, 2016 & 40 & 150 & 0 & 32 & 80 & 3 & (1) (2) (3) (4) (5) \\
\hline $\mathrm{Wu}, 2010$ & 81 & $150-100$ & 0 & 65 & $80-40$ & 2 & (1) (2) (3) (4) \\
\hline $\mathrm{Wu}, 2014$ & 56 & 150 & 5 & 57 & 80 & 9 & (1) (2) (4) \\
\hline $\mathrm{Xu}, 2011$ & 68 & 150 & 0 & 68 & 100 & 3 & (1) (2) (3) (4) (5) \\
\hline Yang, 2017 & 40 & 100 & 0 & 32 & 80 & 3 & (1) (2) (3) (4) \\
\hline Zeng, 2014 & 80 & 50 & 5 & 80 & 30 & 17 & (4) (5) \\
\hline Zhang, 2010 & 60 & 100 & 1 & 68 & 80 & 2 & (4) (5) \\
\hline Zhang, 2013 & 82 & 100 & 0 & 66 & 60 & 1 & (1) (2) (3) (4) \\
\hline Zhou, 2014 & 25 & 200 & 0 & 25 & 100 & 3 & (1) (2) (3) (4) (5) \\
\hline Zhu, 2008 & 60 & 150 & 0 & 50 & 60 & 2 & (1) (2) (4) \\
\hline
\end{tabular}

SI: study index; (1): ALT; (2): AST; (3): T-Bil; (4): adverse effects; (5): curative effect.

and $0.519>0.05$, indicating that the results were not statistically significant. All of them are uniformly distributed in Figure 4. Therefore, no publication bias was found by Begg's test. The results of the literature quality evaluation are shown in Figure 5.
3.3. The Conditions of ADR Development. The specific conditions of the number and symptoms of ADRs in the articles are shown in Table 2.

The overall analytic results of the 24 studies are shown in Figure 6. They all reported ADR development in both the MI 


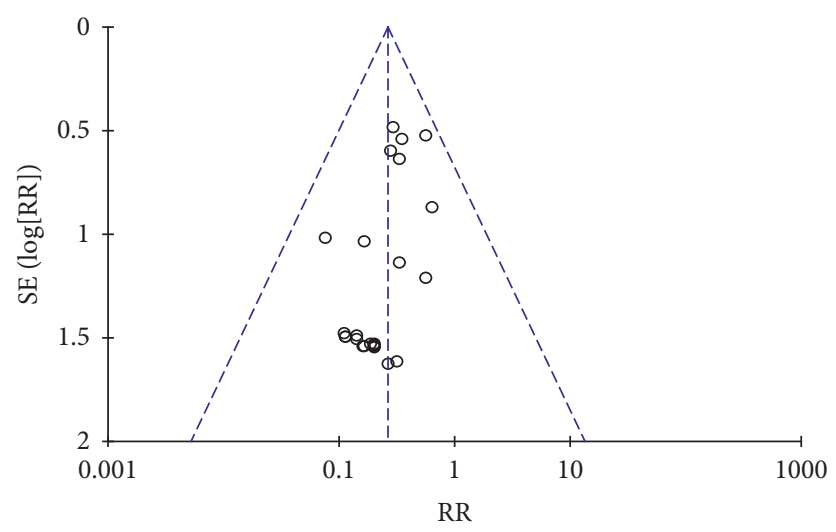

Figure 2: Evaluation of the publication bias of the articles.

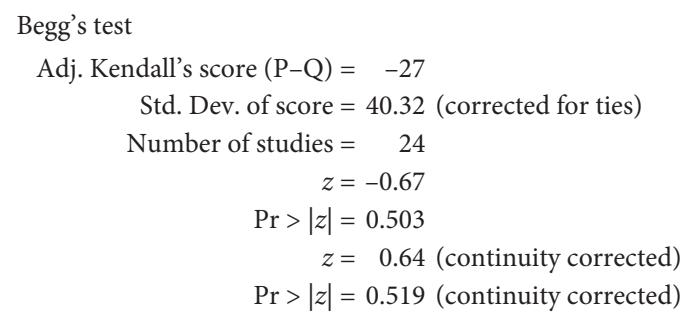

Figure 3: Begg's test.

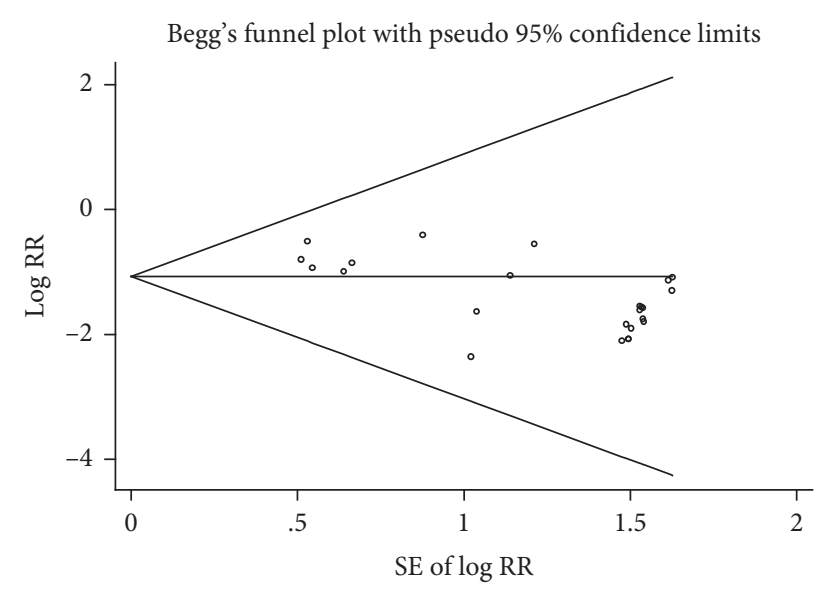

Figure 4: Begg's funnel plot with pseudo 95\% confidence limits.

group and the CG group $(n=1520 / 1237)$. To assess ADR development, the meta-analysis was performed using the fixed-effect model because the heterogeneity among all studies might not be important $\left(I^{2} \leq 50 \%\right)$. The results showed that the RR of ADR development in the MI group was significantly lower than that in the CG group $(R R=0.26$, $95 \% \mathrm{CI}=(0.18,0.38), P<0.00001)$.

\section{Discussion}

The mechanism of ADR development is very complicated. Molecular heterogenicity is one of the reasons for the difference ADR occurrence in glycyrrhizin preparations. $\mathrm{MI}$ is a single-ingredient preparation, in which the non- $8 \alpha-G L$ content is only $1 \%$. CG, as a representative drug of secondgeneration glycyrrhizic acid, mainly contains $18 \beta$-GL and a small amount of $18 \alpha$-GL, and the proportion of $18 \beta-G L$ ranged between $95 \%$ and $99 \%$. This study compared the highpurity $18 \alpha$-GL preparation of MI with the clinically extensively used CG preparation that mainly contained $18 \beta$-GL. Through a comprehensive analysis of the conditions of ADR development in many studies, the results showed that these two preparations had significant differences in the ADR.

4.1. Clinical Presentations of ADRs. The statistical analysis of this study showed that the ADRs of GL primarily manifested as lower limb edema, facial edema, increased blood pressure, 


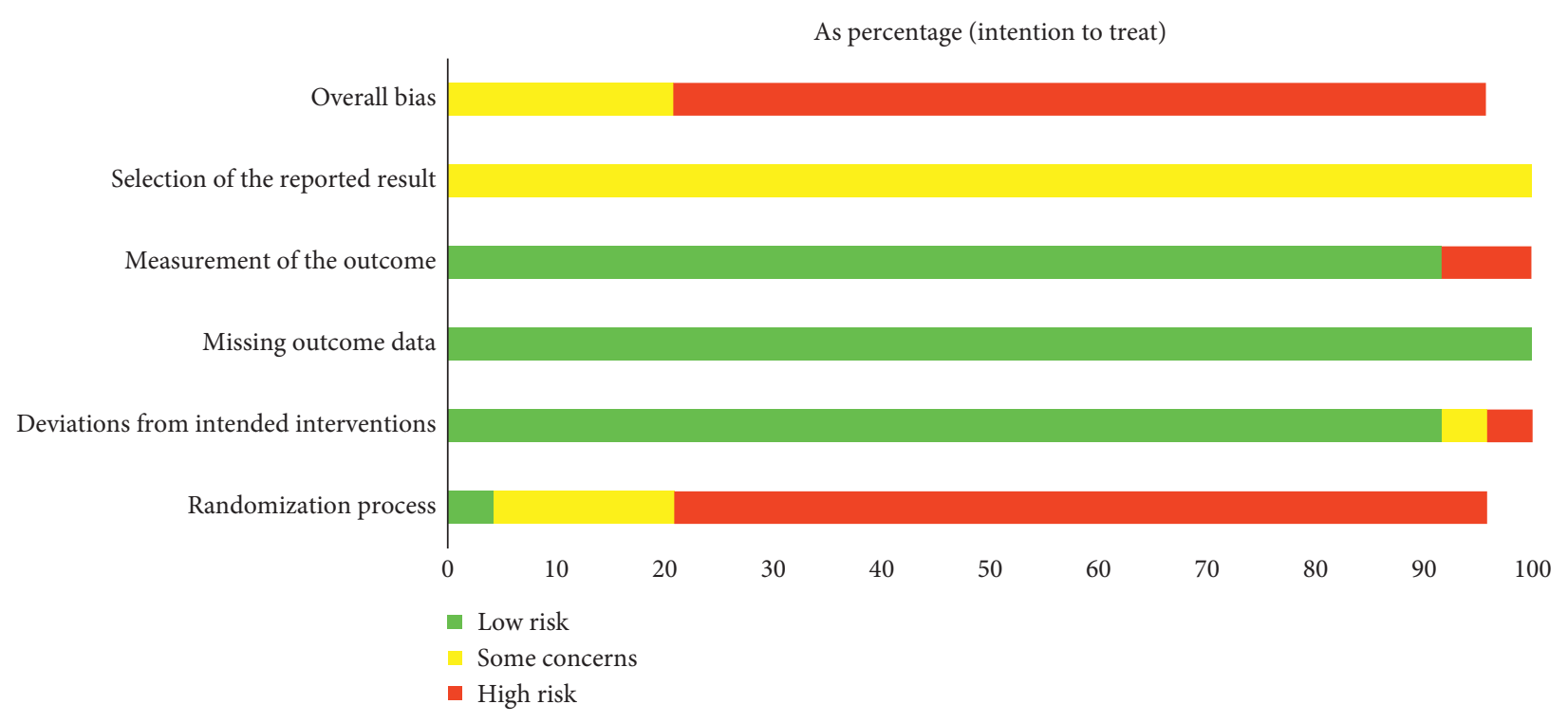

Figure 5: Results of the quality evaluation of the included articles.

TABLE 2: The presentation and number of ADRs in the MI and CG groups.

\begin{tabular}{lcccc}
\hline \multirow{2}{*}{ ADR presentation } & \multicolumn{2}{c}{ MI group } & \multicolumn{2}{c}{ CG group } \\
& Number of ADRs & Proportion (\%) & Number of ADRs & Proportion (\%) \\
\hline Lower limb edema & 3 & 10.71 & 23 & 20.18 \\
Dizziness, headache & 6 & 21.43 & 20 & 18.42 \\
Increased blood pressure & 2 & 7.14 & 13 & 17.54 \\
Palpitation & 5 & 17.86 & 16 & 11.40 \\
Facial edema & 3 & 10.71 & 8 & 7 \\
Gastrointestinal reaction & 5 & 17.86 & 3 & 7.02 \\
Decreased muscle strength & 2 & 7.14 & 0 & 6.14 \\
Hypokalemia & 1 & 3.57 & 1 & 2.63 \\
Hyperkalemia & 0 & 0.00 & 1 & 0.00 \\
Urine occult blood & 0 & 0.00 & 1 & 0.88 \\
Facial flushing & 0 & 0.00 & 114 & 0.88 \\
Skin rash & 1 & 3.57 & 100.00 & 10.88 \\
Total & 28 & & 100.00 \\
\hline
\end{tabular}

palpitation, and dizziness and headache. A total of 112 cases accounted for $78.87 \%$ of the 142 ADR cases. These frequent ADRs were all associated with pharmacological and toxicological effects of GL and were pseudo-aldosteronism reactions. Pseudo-aldosteronism reactions accounted for $67.86 \%$ of ADRs in the MI group and $81.58 \%$ of ADRs in the CG group. With MI as the $18 \alpha$-GL preparation, pseudoaldosteronism was still the main type of ADR reported; however, its incidence was lower than that from the CG presentation, which mainly contained $18 \beta$-GL. These results were consistent with the study of $\mathrm{Li}$ et al. [60].

4.2. The Mechanism of Differences. The current view considers that the pseudo-aldosteronism reaction of GL is associated with the drug itself or its metabolic product [61]. Different structures of GL also have metabolic products with different structures in the body. The metabolic product of $18 \alpha$-GL is $18 \alpha$-glycyrrhetinic acid, and the metabolic product of $18 \beta-\mathrm{GL}$ is $18 \beta$ - glycyrrhetinic acid. Existing preclinical studies have shown that the incidence of pseudo-aldosteronism reactions produced by $18 \alpha$-glycyrrhetinic acid was lower than that produced by $18 \beta$-glycyrrhetinic acid. A possible reason is that $18 \alpha$-glycyrrhetinic acid selectively inhibits type $111 \beta$-hydroxysteroid dehydrogenase, whereas $18 \beta$ glycyrrhetinic acid has inhibitory effects on both type 1 and type $211 \beta$-hydroxysteroid dehydrogenase [4]. These results suggest that differences in the clinical ADRs between these two preparations might also be caused by differences in their molecular conformation. The specific clinical, pharmacological, and toxicological mechanisms and the strength of action require further experimental studies.

4.3. The Influence of the Experimental Design Program. The meta-analysis results were influenced by the quality and number of patients in the included articles. None of 


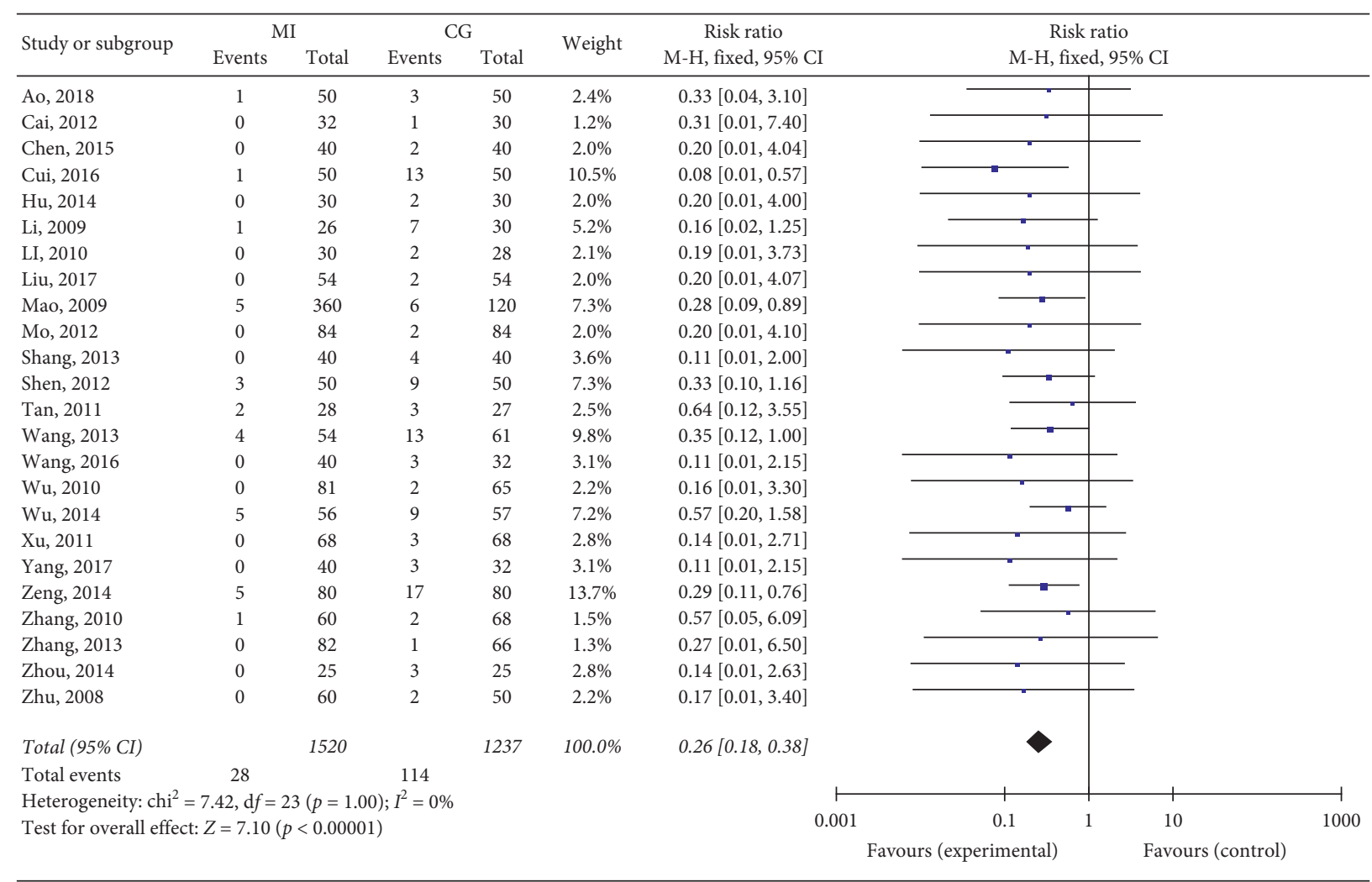

FIgURE 6: Results of the meta-analysis of ADR development.

the articles included in this study described allocation concealment, which might influence the subjective judgments of doctors and patients, particularly the subjective symptoms of ADRs such as dizziness, headache, and palpitation.

\section{Data Availability}

The datasets used and/or analyzed during the present study are available from the corresponding author upon reasonable request.

\section{Conflicts of Interest}

The authors declare that there are no conflicts of interest.

\section{Authors' Contributions}

Hongdou Chen and Menglei Wang participated in the design of the study. Fangfang Zheng and Menglei Wan were involved in writing of the paper and statistical analysis. Other authors participated in the analysis of the literature. All authors read and approved the final manuscript. Hongdou Chen and Fangfang Zheng contributed equally to this work.

\section{Acknowledgments}

This study was supported by Jiangsu provincial six projects of top-notch talent research project (2019 years, LGY2019054) and
Jiangsu Pharmaceutical Association-Tianqing (Lianyungang) Hospital Pharmaceutical Research Project (Q2018080).

\section{Supplementary Materials}

PRISMA statement and evaluation of publication bias of the articles are provided. (Supplementary Materials)

\section{References}

[1] S. Amagaya, E. Sugishita, Y. Ogihara, S. Ogawa, K. Okada, and T. Aizawa, "Comparative studies of the stereoisomers of glycyrrhetinic acid on anti-inflammatory activities," Journal of Pharmacobio-Dynamics, vol. 7, no. 12, pp. 923-928, 1984.

[2] W. B. Ye, T. Z. Lv, and H. Xu, "Clinical efficacy and safety of magnesium iso-glycyrrhizate injection in the treatment of chronic liver disease with elevated alanine aminotransferase (ALT)," The Channel of Pharmaceutical, vol. 31, no. 6, pp. 148-150, 2019.

[3] Y. M. Mao, M. D. Zeng, Y. Chen et al., "Magnesium isoglycyrrhizinate in the treatment of chronic liver diseases: a randomized, double-blind, multi-doses, active drug controlled, multi-center study," Zhonghua Gan Zang Bing Za Zhi, vol. 17, no. 11, pp. 847-851, 2009.

[4] T. Akao, T. Akao, M. Hattori, T. Namba, and K. Kobashi, "Inhibitory effects of glycyrrhetic acid and its related compounds on 3.ALPHA.-Hydroxysteroid dehydrogenase of rat liver cytosol," Chemical \& Pharmaceutical Bulletin, vol. 40, no. 5, pp. 1208-1210, 1992.

[5] J. J. Shuster, "Review: cochrane handbook for systematic reviews for interventions, version 5.1.0, published 3/2011. j. p. 
t. higgins and s. green, editors," Research Synthesis Methods, John Wiley \& Sons, vol. 2, no. 2, , pp. 126-130, Hoboken, USA, 2011.

[6] C. X. Zeng, Q. Yang, and Q. Hu, "Comparative study on the distribution and metabolism of magnesium iso-glycyrrhizate and compound glycyrrhizin in rats," Chinese Pharmacy, vol. 17, no. 20, pp. 1543-1545, 2006.

[7] J. Li, "Risk factors analysis of hypokalemia caused by different glycyrrhizin preparations," Beijing university of traditional Chinese medicine, vol. 98, 2014.

[8] S. F. Song, "Clinical treatment of chronic hepatitis," World's Latest Medical Information Digest (Continuous Electronic Journal), vol. 17, no. 1, pp. 78-90, 2017.

[9] Y. J. Zhu, "24 cases of decompensated cirrhosis treated with magnesium iso-glycyrrhizate," Clinical Medicine, vol. 11, pp. 102-103, 2009.

[10] M. L. Jiang, "Comparative analysis of the efficacy of compound glycyrrhizin and magnesium iso-glycyrrhizate in the treatment of alcoholic hepatitis," Modern Diagnosis and Treatment, vol. 25, no. 19, pp. 4451-4452, 2014.

[11] J. C. Peng, "Comparative study on the efficacy of magnesium iso-glycyrrhizate and compound glycyrrhizin in the treatment of chronic hepatitis," Chinese Modern Doctor, vol. 54, no. 24, pp. 104-106, 2016.

[12] F. P. Meng, H. W. Li, P. Zhao et al., "Clinical observation of magnesium iso-glycyrrhizate in the treatment of chronic hepatitis," Liver, vol. 4, pp. 318-319, 2007.

[13] P. P. Yin and H. Cao, "Application of magnesium isoglycyrrhizate in the treatment of severe hepatitis," Jiangsu Medcine, vol. 35, no. 12, 15.3 pages, 2009.

[14] C. Z. Zhang, "Economic analysis of two glycyrrhizic acid drugs in the treatment of chronic hepatitis b," China Pharmaceutical Industry, vol. 20, no. 6, 56 pages, 2011.

[15] C. H. Jiang, X. H. Wang, and Y. Wang, "Observation on the curative effect of magnesium iso-glycyrrhizate in the treatment of chronic hepatitis," Chinese Modern Doctors, vol. 49, no. 29, pp. 69-70, 2011.

[16] H. L. Liu, M. Y. Yin, S. L. You et al., "Efficacy of glycyrrhizin in the treatment of drug-induced liver damage," Journal of Practical Hepatology, vol. 16, no. 3, pp. 257-258, 2013.

[17] Y. Y. Jiang, H. Wang, P. Xia et al., "Effect of magnesium isoglycyrrhizate on the treatment of unicterous cytomegalovirus hepatitis in infants," Chinese Journal of Medcine, vol. 49, no. 7 , pp. 95-97, 2014.

[18] H. J. Chen, "Comparison of the efficacy of compound glycyrrhizin and magnesium isoglycyrrhizinate in the treatment of alcoholic hepatitis," Trace Element Science of Guangdong, vol. 8, pp. 65-67, 2015.

[19] Y. X. Wang, "Analysis of the effect of magnesium isoglycyrrhizinate and compound glycyrrhizin on chronic hepatitis," Chinese and Foreign Medical Treatment, vol. 34, no. 31, pp. 143-144, 2015.

[20] L. Jiang and X. Yi, "Observation of clinical efficacy of magnesium isoglycyrrhizinate in the treatment of drug-induced liver injury caused by anti-tuberculosis drugs," China Modern Drug Application, vol. 10, no. 5, pp. 117-118, 2016.

[21] L. Mao, C. Y. Feng, and X. Y. Liu, "Observation on the therapeutic effect of glycyrrhizin on viral hepatitis," Journal of Hubei University of Traditional Chinese Medicine, vol. 18, no. 1, pp. 67-69, 2016.

[22] W. W. Pan, "Clinical observation on the treatment of chronic hepatitis B with magnesium isoglycyrrhizinate injection," China Health Standards Administration, vol. 7, no. 10, pp. 1101-1102, 2016.
[23] L. Zhu, "Compound glycyrrhizin injection and magnesium isoglycyrrhizinate injection for the treatment of viral hepatitis with hyperbilirubinemia," World Clinical Medicine, vol. 10, no. 17, pp. 103-104, 2016.

[24] H. Hua, "Clinical effect analysis of compound glycyrrhizin and magnesium isoglycyrrhizinate in the treatment of alcoholic hepatitis," Northern Pharmacy, vol. 14, no. 12, p. 61, 2017.

[25] Y. C. Guan and B. L. Wang, "Comparative analysis of compound glycyrrhizin and magnesium isoglycyrrhizinate in the treatment of alcoholic hepatitis," Clinical Medicine Electronic Journal, vol. 4, no. 71, p. 14011, 2017.

[26] F. Jiang, "A comparative study of the therapeutic effect of magnesium isoglycyrrhizinate and compound glycyrrhizin on chronic hepatitis," Chinese Continuing Medical Education, vol. 9, no. 14, pp. 201-202, 2017.

[27] S. C. Yin, "Observation of therapeutic effect of 70 cases of chronic hepatitis B treated with magnesium isoglycyrrhizinate injection," Jilin Medicine, vol. 38, no. 9, pp. 1728-1729, 2017.

[28] J. M. Wu, "Observation of the therapeutic effect of magnesium isoglycyrrhizinate injection on chronic hepatitis," Zhejiang Clinical Medicine, vol. 19, no. 6, pp. 1076-1077, 2017.

[29] Y. Liu, "Observe the clinical efficacy of three glycyrrhizin preparations of compound glycyrrhizin, diammonium glycyrrhizinate, and magnesium isoglycyrrhizinate in the treatment of viral hepatitis with hyperbilirubinemia," Chinese Medical Guide, vol. 16, no. 9, pp. 175-176, 2018.

[30] M. Ao, S. Y. Chen, and Y. L. . Wu, "A comparative study on the efficacy of compound glycyrrhizin and magnesium isoglycyrrhizinate in the treatment of chronic hepatitis to reduce transaminase," Family Medicine, vol. 8, pp. 144-145, 2018.

[31] Z. Y. Huang, "Analysis of clinical effect of magnesium isoglycyrrhizinate injection on impaired liver function," China Health Nutrition, vol. 17, no. 17, p. 346, 2019.

[32] M. J. Wang, L. Li, L. Z. Wang et al., "Clinical effect analysis of magnesium isoglycyrrhizinate in the treatment of autoimmune hepatitis," Community Medical Journal, vol. 14, no. 1, pp. 53-54, 2016.

[33] S. S. Ouyang, "Observation of efficacy and safety evaluation of 100 cases of hepatic disease treated with magnesium isoglycyrrhizinate injection," Modern Health, vol. 14, pp. 157-158, 2014.

[34] Y. D. Guo, "Comparing the clinical effect of magnesium isoglycyrrhizinate and compound glycyrrhizin on chronic hepatitis," China Health Standards Management, vol. 7, no. 24, pp. 102-103, 2016.

[35] Y. Nie, "Observation of the effect of magnesium isoglycyrrhizinate injection on severe hepatitis," Modern Medicine and Clinical, vol. 29, no. 1, pp. 55-58, 2014.

[36] J. J. Zhi, "Therapeutic effect of magnesium isoglycyrrhizinate on autoimmune hepatitis," China Practical Medical Journal, vol. 45, no. 12, pp. 110-114, 2018.

[37] H. N. Liu, Y. Wang, and H. Y. Guo, "Comparative study on the clinical efficacy of different kinds of glycyrrhizin in the treatment of chronic hepatitis," Journal of Chronic Diseases, vol. 18, no. 9, pp. 1027-1029, 2017.

[38] L. Y. Zhou, "Clinical analysis of compound glycyrrhizin and magnesium isoglycyrrhizinate in treating alcoholic hepatitis," Medicine and Health, vol. 22, no. 2, pp. 18-19, 2014.

[39] L. J. Chen, "Comparison of the efficacy of magnesium isoglycyrrhizinate and compound glycyrrhizin in the treatment of chronic hepatitis," Modern Health, vol. 24, p. 489, 2015. 
[40] J. Zhu and Z. Y. Gao, "Clinical observation of magnesium isoglycyrrhizinate in the treatment of chronic hepatitis," Tianjin Pharmaceuticals, vol. 3, pp. 39-41, 2008.

[41] S. P. Zeng, "Discussion on the clinical effect of magnesium isoglycyrrhizinate on eczema," Contemporary Medicine, vol. 20, no. 32, pp. 141-142, 2014.

[42] J. Tan and M. Zhou, "Observation of the therapeutic effect of magnesium isoglycyrrhizinate on chronic hepatitis," Strait Pharmaceutical, vol. 23, no. 5, pp. 199-200, 2011.

[43] Q. J. Xu and Z. K. He, "Clinical study of magnesium isoglycyrrhizinate in the treatment of chronic hepatitis," Clinical Medicine, vol. 31, no. 7, pp. 74-75, 2011.

[44] J. B. Wu, S. R. Ma, W. H. Huang et al., "Comparison of the efficacy of magnesium isoglycyrrhizinate and compound glycyrrhizin in the treatment of non-alcoholic fatty liver," China Pharmaceutical Industry, vol. 23, no. 7, pp. 64-65, 2014.

[45] T. Li, L. Chen, and C. Guo, "Comparison of clinical efficacy of $\alpha$-body glycyrrhizic acid and $\beta$-body glycyrrhizic acid in the treatment of viral hepatitis," Modern Chinese Medicine, vol. 3, no. 18 , pp. 119-120, 2009.

[46] J. Zhang, X. Y. Liu, and Y. Wang, "Observation of the clinical effect of magnesium isoglycyrrhizinate in the treatment of chronic hepatitis," Shanxi Medical Journal (Second Half Monthly), vol. 42, no. 6, pp. 661-663, 2013.

[47] C. H. Wang, S. J. Zheng, and G. Q. Zhan, "Comparison of the therapeutic effect of magnesium isoglycyrrhizinate and compound glycyrrhizin on chronic hepatitis," Journal of Clinical Gastroenterology, vol. 425, no. 4, pp. 203-205, 2013.

[48] P. J. Shang, "Comparative analysis of compound glycyrrhizin and magnesium isoglycyrrhizinate in the treatment of alcoholic hepatitis," Chinese Disability Medicine, vol. 21, no. 8, pp. 257-258, 2013.

[49] L. Q. Cai, Y. L. Wu, and J. H. Zhan, "Comparison of the efficacy of two glycyrrhizin preparations in the treatment of chronic hepatitis B," Journal of Pharmaceutical Epidemiology, vol. 21, no. 4, pp. 160-161, 2012.

[50] Q. F. Shen, "Comparison of the efficacy of different glycyrrhizic acid drugs in the treatment of chronic hepatitis," Channel Pharmaceuticals, vol. 24, no. 7, pp. 101-103, 2012.

[51] Y. C. Cui, "Comparison of the efficacy of magnesium isoglycyrrhizinate and compound glycyrrhizin in the treatment of alcoholic hepatitis," World's Latest Medical Information, vol. 16, no. 79, p. 211, 2016.

[52] R. R. Mo, M. Z. Zhou, and Y. Q. He, "Comparison of the efficacy of magnesium isoglycyrrhizinate and compound glycyrrhizin in the treatment of chronic hepatitis," China Contemporary Medicine, vol. 19, no. 30, pp. 82-83, 2012.

[53] Y. C. Wu, "Observation of the clinical effect of magnesium isoglycyrrhizinate on chronic hepatitis," Journal of Practical Liver Disease, vol. 13, no. 1, pp. 34-36, 2010.

[54] C. Z. Zhang, Y. Wang, and G. L. Qiu, "Comparison of the efficacy of different glycyrrhizic acid drugs in the treatment of chronic hepatitis," China Pharmaceutical, vol. 19, no. 11, p. 83, 2010.

[55] C. W. Wang and P. Zhou, "Observation of the clinical effect of magnesium isoglycyrrhizinate in the treatment of abnormal liver function," China Practical Medicine, vol. 11, no. 3, pp. 133-134, 2016.

[56] J. K. Hu, H. B. Gao, J. R. Zhu et al., "Observation and economic analysis of the therapeutic effect of magnesium isoglycyrrhizinate on anti-rheumatic drug-induced liver damage," Medical Information, vol. 24, pp. 95-96, 2014.

[57] Q. B. Li, "Analysis of 30 cases of cholestatic hepatitis treated with magnesium isoglycyrrhizinate combined with adenosylmethionine," Journal of Chinese Physician Training, vol. 1133, no. 7, pp. 58-59, 2010.

[58] S. Yang and Z. Y. Huang, "Observation of clinical effect of magnesium isoglycyrrhizinate on abnormal liver function," Health Wenhui, vol. 7, p. 17, 2017.

[59] M. Ao, S. Y. Chen, Y. L. Wu et al., "Comparative study of compound glycyrrhizin and isoglycyrrhizin in the treatment of chronic hepatitis to reduce bilirubin," Diet Health, vol. 5, no. 31, p. 71, 2018.

[60] Z. Q. Li Zhiqiang, C. Xia, Y. Wang, G. Wang, and J. Shi, “A systematic review of the effectiveness and safety of magnesium isoglycyrrhizinate injection in comparison with four commonly used drugs in treating drug-induced liver damage," Chinese Pharmacy, vol. 26, no. 33, pp. 4678-4681, 2015.

[61] L. Wl, J. L. Zhang, Y. Dong et al., "Advances in research on the action and drug interaction of glycyrrhizic acid and glycyrrhetinic acid pseudoaldosterone," Journal of Liaoning University of Traditional Chinese Medicine, vol. 20, no. 10, pp. 216-220, 2018. 